\title{
Competence of Nurses Relating Self-Directed Learning in Saudi Arabia: A Meta-Analysis
}

\author{
Abdulaziz M. Alsufyani ${ }^{1}$, Ahmad E. Aboshaiqah², Mahaman L. MOUSSA ${ }^{2}$ \\ Omar G. Baker ${ }^{2} \&$ Khalid E. Almalki ${ }^{3}$ \\ ${ }^{1}$ Comprehensive Rehabilitation Center, Ministry of Labour and Social Development, Taif, Saudia Arabia \\ ${ }^{2}$ College of Nursing, King Saud University, Riyadh, Saudi Arabia \\ ${ }^{3}$ Primary Healthcare Center, Ministry of health, Riyadh, Saudi Arabia \\ Correspondence: Mahaman L. Moussa, College of Nursing, King Saud University, Riyadh, P.O. BOX 642, 11421, \\ Saudi Arabia. E-mail: mmoussa@ksu.esu.sa
}

Received: January 10, 2019 Accepted: July 23, 2019 Online Published: July 30, 2019

doi:10.5539/gjhs.v11n9p145 URL: https://doi.org/10.5539/gjhs.v11n9p145

\begin{abstract}
Self-directed learning (SDL) has become important for medical students for developing their independent learning skills. This may help them in enhancing their sense of assertiveness and responsibility. Therefore, the present study aims to evaluate the competency based learning among the nurses as it is important to secure and promote the nursing services. The study has conducted a broad spectrum of research studies between the 10 -year duration i.e., between the time-period of (2009-2018). The studies shedding light on details of the competence of nurses' relation to SDL in Saudi Arabia were reviewed. The results have shown significant post-intervention enhancements with a pooled random-effects standardized mean difference of 0.81 . The effect size of learning was higher as compared to the reaction with respect to the assessment level. It has also been shown that students with medium-competence simulation have higher effect size as compared to high-competence simulation and low-competence simulation.
\end{abstract}

Keywords: competence, nurses, self-directed learning, Saudi Arabia

\section{Introduction}

Self-Directed Learning is a natural process that requires essential skills in both the educational and practical world. Students can be highly motivated by using this learning approach as they are the planners for their own knowledge. Due to this, they can experience the sense of independence while learning (Jaleel \& OM, 2017). In line with Saxena (2013), students are always found to be engaging in the learning activities because of the SDL process. They have to seek for knowledge on their own and have to apply that knowledge along with their skills. This approach provides solutions to the problems they face in their learning setting and allows to be encouraged for their life-long learning.

With a rapid emergence of SDL process in the past few years, it is known to be an educational technique that is being used in the tertiary institutions. Not only students but the medical professionals take responsibility for their own learning and decision making. They identify the learning goals and evaluate the resources used in the learning process (El-Gilany \& Abusaad, 2013). SDL is a process in which students and professionals take complete control and self-determine to identify the beneficial aspects. However, the amount of control they want to have is solely based on their aptitude, personality characteristics and assertiveness (du Toit-Brits \& van Zyl, 2017; Davis, 2015). Due to the promotion by the several institutions, SDL seems to be essential as it provides value to the development of the professionals and these professionals can become life-longer learners. SDL is being embraced globally in the field of medical so that the professionals as well as medical students can achieve the skills. Its purpose is to continuously polish themselves by using the relevant knowledge and skills in the world of medicine (Premkumar et al., 2013).

When it comes to the health care professionals such as nurses, they seem to work in the multifaceted professional setting. They continuously face challenges that are being created from social and scientific changes occurred in the field of healthcare due to the lack of SDL in the nursing competency. Nursing competency is based on the skills, behaviors, knowledge, and ability that nurses possess to perform all the assigned tasks skillfully (Yang \& Jiang, 
2014). Self-Directed Learning Readiness (SDLR) seems to be vital for the better academic and professional performance. Hence, it is explicitly stated that SDL is important as it can have a great influence on the nursing competency. Despite of the importance on SDL, a very few evidence-based data can be found and its relationship with nursing competency (Yang \& Jiang, 2014; Van Rensburg \& Botma, 2015). Moreover, it is also observed that nurses do not possess competency in terms of SDL in their professional setting. Due to this, majority of the nurses experience high level of anxiety when it comes to direct their own learning and skills (Van Rensburg \& Botma, 2015).

In the continuously changing environment, SDL seems to be vital for the medical students and professionals so that they can develop their independent learning skills. Due to this, they can enhance their sense of responsibility and assertiveness. These are the key attributes for the medical professionals in their career. To this end, the present study is focusing on the competency based learning among the nurses as it is important to secure and promote the nursing services. Increased nursing competency can bring a positive change in the professional settings as it increases the confidence level and satisfaction in their practices. It also provides assistance to the nurses to reduce their frustration level in the clinical setting when they are confronted with a confusion about what was learned in the classroom and what is the need right now. Therefore, SDL is required to be introduced in the educational as well as professional settings to make nurses learn, engage them and maximize their nursing competencies in their clinical practices. Additionally, SDL is being observed to have a direct influence on the nursing competency. The present study explores the competence of nurses relating Self-Directed Learning in Saudi Arabia using a Meta-analysis technique.

\section{Materials and Methods}

An extensive literature review has been conducted using a broad spectrum of research studies, primarily taken from; ERIC, Google Scholar, PubMed, and Medline for authentic and quality studies. The studies chosen for the literature discussed the competence of nurses relating Self-Directed Learning in Saudi Arabia. Only research and review articles were exclusively taken into consideration for the study and the rest of the categories were excluded. Only the studies which were published between the 10-year duration i.e., between the time-period of (2009-2018) were considered for the study.

Meta-analysis was conducted along with a set of statistical techniques, which were utilized to review the independent studies based on the specific area of research. Meta-analysis was used to draw narrative discussions, which further elaborate the topic of research. There were five phases which were followed during the meta-analysis; (a) Identification of the relative studies; (b) Determination of the inclusion criteria; (c) Coding; (d) Extraction of the calculations and data and (e) Analysis of data.

All the mentioned platforms were extensively researched for the literature search. Primarily, based on the keywords and keeping in mind the inclusion and exclusion criteria for the study, 1750 abstracts were searched and retrieved for the study. At first, each abstract was investigated by an investigator along with a professional. Due to less reliability and authenticity, the older versions, case reports, blogs, and essays have been excluded from the study. Those studies which were based on cross-sectional, prospective and retrospective analysis were included in the study. These studies shed light on the details of the competence of nurses relating Self-Directed Learning in Saudi Arabia.

Extensive scrutiny of the abstracts was carried out to select only the most relevant articles for the study. Those articles that exclusively deal with the competence of nurses relating Self-Directed Learning in Saudi Arabia were promoted. The first phase has excluded almost 1550 abstracts and the remaining articles were further processed for the second phase of scrutiny. In total, only 150 articles have been selected for the study to conduct in-depth analysis of the topic of research. Afterwards, strict inclusion criteria were applied to the selected studies, which resulted in the retrieval of only 25 articles, after the elimination of 125 studies. The remaining 25 articles were evaluated on the basis of conceptual framework to assure the significance and the direction of the study. In the next step, 18 articles were further eliminated due to reduced relevance with the topic and the remaining 7 articles were used for the systematic review analysis. A pictorial depiction has been presented in Figure 1. 


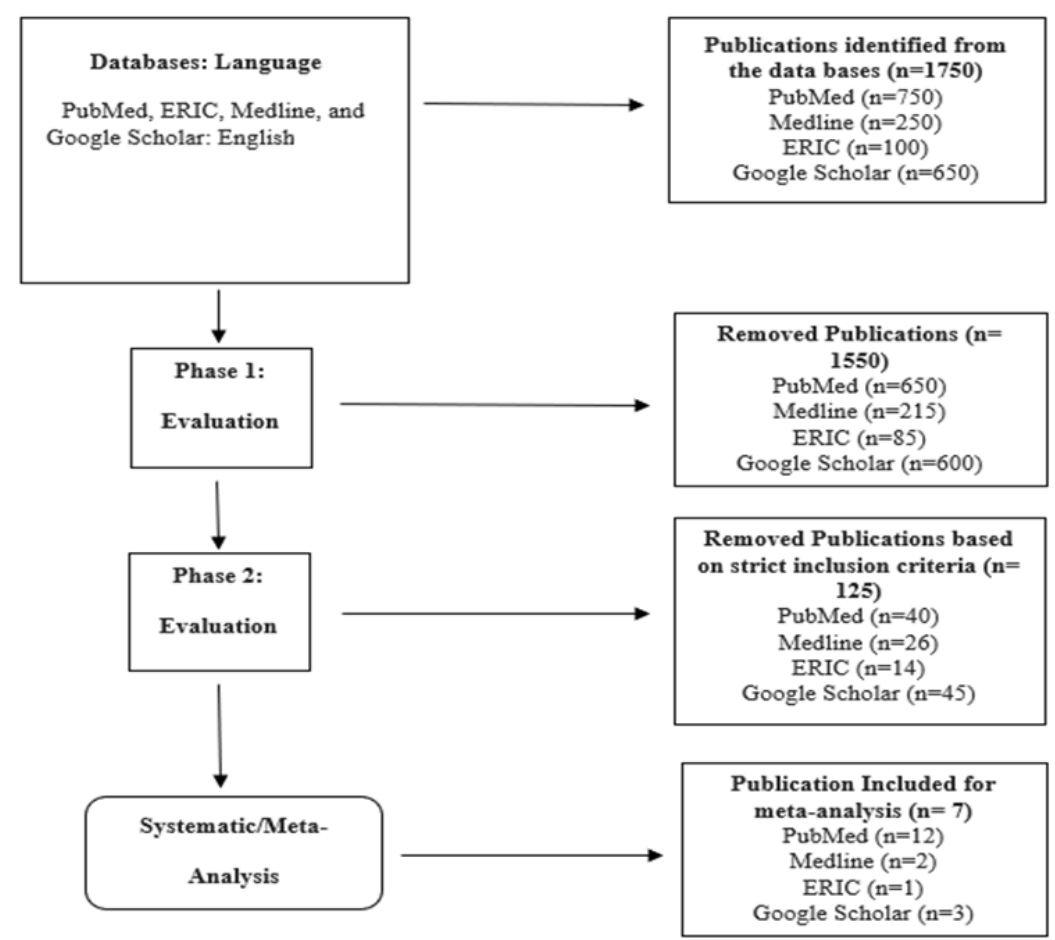

Figure 1. Literature Search Process

\section{Results}

A total of 1750 studies were found in the selected databases using a cross-reference strategy. In contrast, a total of 1550 duplicates were excluded and 125 studies were comprehensively assessed. A total of 7 studies were selected that comprised the final sample of nursing competence towards self-directed learning in Saudi Arabia after evaluation of the methodological quality. The selected studies were published in English and had a level of evidence II based on the classification of quantitative studies. These studies were conducted in China and the US in the article format.

Table 1 has shown the Meta-analysis for 7 selected studies based on the effect size and standard error. The findings have indicated that there was heterogeneity when these studies combined in a review process $(\mathrm{Q}=135.00, \mathrm{p}<.01)$. The findings have indicated significant post-intervention enhancements with a pooled random-effects standardized mean difference of 0.81 .

Table 1. Overall findings of the meta-analysis via random effect model

\begin{tabular}{lllll}
\hline $\mathbf{N}$ & $\mathbf{- 9 5 \%} \mathbf{C I}$ & $\mathbf{E S}$ & $\mathbf{+ 9 5 \%} \mathbf{C I}$ & $\mathbf{S E}$ \\
\hline $\mathbf{7}$ & 0.43 & 0.81 & 0.95 & 0.19 \\
\hline
\end{tabular}

$\mathrm{N}=$ number of studies, $\mathrm{SE}=$ standard error, $\mathrm{ES}=$ Effect size.

Table 2 has identified the precision of each study based on the size of the confidence interval. The effectiveness of self-directed learning has been indicated from the findings of each study with squares toward the left side. On the contrary, the findings on the right side indicated the self-directed learning to be less effective among nursing students. 
Table 2. Size of the confidence interval

\begin{tabular}{lllll}
\hline \multirow{2}{*}{ Study name } & Statistics for each study & & \\
\cline { 2 - 5 } & Standard difference in means & Lower limit & Upper limit & p-value \\
\hline White (2013) & 0.514 & 0.377 & 0.852 & 0.001 \\
Thomas (2012) & 1.117 & 1.497 & 1.638 & 0.000 \\
Parker (2011) & 0.132 & -0.115 & 0.390 & 0.103 \\
Smith (2013) & 0.134 & -0.214 & 0.583 & 0.303 \\
Weiner (2011) & 0.234 & -0.053 & 0.554 & 0.115 \\
Andrighetti (2011) & 1.115 & 0.271 & 1.048 & 0.003 \\
Alfes (2011) & 0.294 & 0.129 & 0.570 & 0.003 \\
\hline
\end{tabular}

Dependency of study outcomes was presented in Table 3 as there were multiple findings present within a study. A sub-group analysis was performed using a random effects model as homogeneity tests demonstrated that each sub-group was heterogeneous. The analysis was based on domain of learning, evaluation methods, and level of assessment. The effect sizes were higher for performance-based evaluation as compared to examinations, grades, and self-assessed evaluation with respect to type of evaluation. In addition, the effect size of learning was higher as compared to the reaction with respect to the assessment level. The effect size of psychomotor was higher as compared to cognitive and affective with respect to the learning domain.

Table 3. Effect sizes based on evaluation and evidence levels by sub-group

\begin{tabular}{lllllll}
\hline Consequences & Sub-categories & $\mathbf{K}$ & $\mathbf{- 9 5 \%} \mathbf{C I}$ & $\mathbf{E S}$ & $\mathbf{+ 9 5 \%} \mathbf{C I}$ & $\mathbf{S E}$ \\
\hline \multirow{3}{*}{ Domain of learning } & Cognitive & 11 & 0.02 & 0.33 & 0.65 & 0.13 \\
& Psychomotor & 23 & 0.54 & 0.84 & 1.12 & 0.11 \\
& Affective & 18 & 0.35 & 0.73 & 1.01 & 0.11 \\
\hline \multirow{3}{*}{ Level of evidence } & Grade & 1 & -0.45 & 0.11 & 0.81 & 0.23 \\
& Performance-based & 21 & 0.76 & 1.12 & 1.35 & 0.11 \\
& Examination & 7 & 0.03 & 0.35 & 0.61 & 0.13 \\
\hline \multirow{2}{*}{ Assessment level } & Self-evaluated & 29 & 0.21 & 0.45 & 0.65 & 0.05 \\
\hline & Reaction & 5 & 0.25 & 0.35 & 0.55 & 0.15 \\
& Learning & 55 & 0.45 & 0.64 & 0.80 & 0.05 \\
\hline
\end{tabular}

Table 4 has presented effect sized on the basis of learning environments. The findings have shown higher effect size of clinical course as compared to foundation course and legal ethical course with respect to course name. With respect to the student's level, findings have indicated that students in $3^{\text {rd }}-4^{\text {th }}$ year have higher effect size as compared to $1^{\text {st }}$ year students, $2^{\text {nd }}$ year students, and nurses. With respect to competence level, students with medium-competence simulation have higher effect size as compared to high-competence simulation and low-competence simulation. 
Table 4. Effect Sizes based on Learning Environments by Subgroup

\begin{tabular}{lllllll}
\hline Subgroup & Categories & $\mathbf{K}$ & $\mathbf{- 9 5 \%} \mathbf{C I}$ & $\mathbf{E S}$ & $\mathbf{+ 9 5 \%} \mathbf{C I}$ & $\mathbf{S E}$ \\
\hline \multirow{3}{*}{ Course name } & Clinical & 5 & 1.16 & 1.76 & 1.46 & 0.20 \\
& Foundation & 1 & -0.58 & 0.20 & 1.39 & 0.50 \\
& Legal ethical & 1 & -0.58 & 0.85 & 1.39 & 0.11 \\
\hline \multirow{3}{*}{ Level of competence } & HCS & 34 & 0.57 & 0.71 & 1.14 & 0.07 \\
& MCS & 1 & 0.89 & 1.82 & 2.76 & 0.37 \\
& LCS & 11 & 0.40 & 0.24 & 0.51 & 0.11 \\
\hline \multirow{3}{*}{ Student level } & $1^{\text {st }}$ year & 1 & -0.14 & 0.38 & 1.11 & 0.23 \\
& $2^{\text {nd }}$ year & 4 & 0.03 & 0.35 & 0.81 & 0.11 \\
& $3^{\text {rd }}-4^{\text {th }}$ year & 11 & 0.41 & 0.76 & 1.15 & 0.13 \\
& Nurse & 11 & 0.11 & 0.23 & 0.44 & 0.19 \\
\hline
\end{tabular}

$\mathrm{HCS}=$ high-competence simulation; $\mathrm{MCS}=$ medium-competence simulation; $\mathrm{LFS}=$ low-competence simulation; $\mathrm{k}=$ number of effect size, $\mathrm{ES}=$ effect size; $\mathrm{SE}=$ standard error.

\section{Discussion}

The present study has addressed the competency of Saudi nurses regarding SDL based on their demographic characteristics. The results have depicted satisfactory level of readiness and willingness among the nurses towards SDL. The analysis shown in the present study is I $\mathrm{n}$ support with previous studies conducted in other Saudi universities (El-Gilany \& Abusaad, 2013; Shahin \& Tork 2013). The study results have shown that majority of the nurses are featured as independent learners and are provided with the chance to address the teaching opportunities through SDL available among the Saudi nursing students.

A study conducted by White et al. (2013) compared the confidence and cognitive skills between senior students divided into two groups. The results depicted that the use of high-fidelity simulation was not capable of enhancing the cognitive skills and level of confidence among the nurses. Another similar study conducted by Thomas (2012) compared the confidence level among nursing students provided with high-fidelity simulation course and traditional clinical experience. The results of the study were consistent with the present study as it showed increased self-confidence of the students after experiencing clinical simulation. The simulated experiences are likely to enhance the clinical practicum experience of the nursing students. These experiences might range from a simple task of using task trainer or a complex task of adjusting human patient simulators. The nursing schools may seek opportunities for collaborating with other nursing schools with the wave of simulation ripping across attaining nursing education and the associated costs.

These results are congruent with the findings of Chinese study (Yuan et al., 2012), Nepalese study and two Australian studies (Williams et al., 2013; Smedley, 2007). These studies have affirmed the elevated levels of readiness for SDL among the undergraduate nursing students. It has also been shown that undergraduate nursing students are not well-suited to interactive teaching strategies because of poor foundation in some general schools in different countries. Moreover, it has also been shown that problem based learning (PBL) and independent learning activities enabled the nursing students to use their learning abilities.

In the community-bases setting, it is important for the nurses to prepare the future nurses for the increase in number of elderly individuals. A similar study conducted by Smith (2013) indicated that nursing students were satisfied with the geriatric home care simulation experience and it has positive impact on their level of confidence. Therefore, it can be stated that it would be significant to develop geriatric simulation education experience for the new graduated nurses. It has been shown that self-directed education program has the ability to shift acquisition of skills and allow the instructors to facilitate low-fidelity simulation and debriefing (Weiner, 2011). This may result in significant improvement in educational efficiency of neonatal resuscitation course with significant decrease in the course duration.

The analysis conducted in the present study has depicted that the participants had moderate level on the self-management domain. This finding was consistent with the local study conducted in Saudi Arabia by Shahin \& Tork, (2013), and to overseas study conducted in Pakistan (Said et al., 2015). The desire for learning subscale is designed to determine the learner's openness, needs, and willingness for learning as well as to which extent is the 
learner self-evaluated. However, the overall results showed the current study's participants demonstrated a modest perception towards the desire for learning subscale. Therefore, that positive outcomes of SDL could explain the highest perception toward desire for learning domain among nursing students.

A study conducted by Andrighetti (2011) aimed to investigate the efficiency of high-fidelity simulations concerned with obstetric emergencies to manage complications like shoulder dystocia and postpartum hemorrhage. The results showed positive impact of high-fidelity simulations among the students nursing students by increasing their level of confidence in managing shoulder dystocia and postpartum hemorrhage. Another similar study conducted by Alfes (2010) emphasized on the implementation of fidelity simulations promoting self-confidence and satisfaction with learning among the nursing students.

\section{Implications - Differentiating Between SDL and PBL}

The inclusion of learner involvement, improvement of self-directed learning, and collaborative faculty-student relationships in self-directed learning promotes the learning experience of nurses. Precisely, self-directed learning strategy and critical thinking is associated for accumulating the knowledge of learners as well as to encourage and emphasize on the development of learners toward particular competencies and skills. Nurses have opportunities to advance their sense of responsibility for their own growth in which they are stimulated to think critically during the process of self-directed learning-critical thinking. Nurses take part in self-reflection and reflection on peers in the tutorial procedure in every aspect of possible discussions. Nurses are encouraged for presenting their constructive criticism, the tutor, and their peers in the skill areas of self-directed learning, independent study, communication, interaction, creativity, and reasoning within the dynamic group process.

It is important for teachers to act as facilitators as they encourage learners toward self-directed learning and assure that all learners are involved in the process. Learners cannot transmit expert knowledge to other learners but increase their knowledge by inquiring questions and stimulating particular kinds of cognitive activities. Self-directed learning needs learners to invest more energy and time to conduct research, share information, comment on information shared by their peers, and search for information. The learning social environment and self-regulation procedures are included as important ingredients of the success in self-directed learning among undergraduate nursing students. The results of the systematic review can allow nursing educators for improving opportunities for focusing on designing curriculum and clinical reasoning that include self-directed learning. In this regard, the focus of facilitators should be on the implementation of self-directed strategies for nurses to meet goals for improving clinical reasoning as well as collaboration skills.

From the meta-analysis, it has been emphasized that self-directed learning skills of students were significantly linked with the preference to seek a graduate degree as compared to those who did not have a similar preference. In this perspective, high motivation levels can be achieved by self-directed learners as they are motivated for the self-development and intended to maintain their education. The preference to achieve a graduate education might be increasing through these qualities, which consequently lead to academic development and professional development. Another reason for such motivation is that it allows nurses to excel in their fields, maintain their learning, have in-depth knowledge, and develop themselves. Additionally, nurses seeking graduate education can often appreciate learning and are encouraged to learn.

The findings of the meta-analysis have indicated that instructional environments should be devised for improving self-control skills of nurses, considering enhanced practices. In addition, these environments can involve the use of learning performance assessment scales, cognitive or upper-cognitive learning strategies, and reflective journals. Moreover, it allows facilitators and educational instructors for developing creative thinking skills among students, initiating from elementary school. Facilitators should identify their opinions and their own learning needs to identify learning objectives as well as to achieve higher self-directed learning skills among nurses. Several learning strategies should be discussed and adopted in classes, and students should be stimulated for monitoring and assessing their own learning procedures. Lastly, special learning experiences should be used for helping students to become critical thinkers by improving their self-educated learning skills.

\section{Conclusion}

The present study has the competence of nurses relating SDL in Saudi Arabia using a meta-analysis technique. The results showed significant enhancement of post-intervention scores with a pooled random-effects standardized mean difference of 0.81 . The study analysis was based on domain of learning, evaluation methods, and level of assessment. The results have also depicted higher effect size of clinical course as compared to foundation course and legal ethical course with respect to course name. It is prudent to nursing educators to continually appraise the students' preferred learning strategy and learning needs to increase the success opportunity of the learning 
outcomes. The availability of the relevant learning resources is among the most important techniques which serve the interactive learning strategies. However, the future studies need to include a large number of studies conducted recently to investigate different perceptions regarding SDL.

\section{Acknowledgements}

The author would like to thank King Abdulaziz City for Science and Technology (KACST) funding and supporting this research through the initiative of KACST for Graduate Students Research Program (GRANTS).

\section{Competing Interests Statement}

The authors declare that there are no competing or potential conflicts of interest.

\section{References}

Alfes, C. M. (2011). CM evaluating the use of simulation with beginning nursing students. J. Nurs. Educ., 50(2), 89-93. http://dx.doi.org/10.3928/01484834-20101230-03

Andrighetti, T. P., Knestrick, J. M., Marowitz, A., Martin, C., \& Engstrom, J. L. (2012). Shoulder dystocia and postpartum hemorrhage simulations: student confidence in managing these complications. J. Midwifery Womens Health, 57(1), 55-60. http://dx.doi.org/ 10.1111/j.1542-2011.2011.00085.x

Davis, J. (2015). Education through self-directed learning. Aust Nurs Midwifery J., 23, 26-27.

Du Toit-Brits, C., \& van Zyl, C. M. (2017). Self-directed learning characteristics: making learning personal, empowering and successful. Africa Education Review, 14(3-4), 122-141.

El-Gilany, A., \& Abusaad, F. E. (2013). Self-directed learning readiness and learning styles among Saudi undergraduate nursing students. Nurse Education Today, 33(9), 1040-1044. http://dx.doi.org/10.1016/j.nedt.2012.05.003

Jaleel, S., \& OM, A. (2017). A Study on the Relationship between Self Directed Learning and Achievement in Information Technology of Students at Secondary Level. Universal Journal of Educational Research, 5(10), 1849-1852.

Parker, R. A., McNeill, J. A., Pelayo, L. W., Goei, K. A., Howard, J., \& Gunter, M. D. (2011). Pediatric clinical simulation: a pilot project. J. Nurs. Educ., 50(2), 105-111. http://dx.doi.org/10. 3928/01484834-20101230-05

Premkumar, K., Pahwa, P., Banerjee, A., Baptiste, K., Bhatt, H., \& Lim, H. J. (2013). Does medical training promote or deter self-directed learning? A longitudinal mixed-methods study. Academic Medicine, 88(11), 1754-1764.

Said, A. B., Ghani, N., Khan, A., \& Kiramat, M. (2015). Examination of Self-Directed Learning Readiness among Baccalaureate Nursing Students in Peshawar Pakistan. International Journal of Innovative Research \& Development, 4(2), 257-262.

Saxena, S. (2013). How technology supports self-directed learning. EdTech Review.

Shahin, E. S., \& Tork, H. M. (2013). Critical thinking and self-directed learning as an outcome of problem-based learning among nursing students in Egypt and Kingdom of Saudi Arabia. Journal of Nursing Education and Practice, 3(12), 103-110. http://dx.doi.org/10.5430/jnep.v3n12p103

Smedley, A. (2007). The self-directed learning readiness of first year bachelor of nursing students. Journal of Research in Nursing, 12(4), 373-385. http://dx.doi.org/10.1177/1744987107077532

Smith, S.J., \& Barry, D. G. (2013). An innovative approach to preparing nursing students for care of the elderly in the home. Geriatr. Nurs., 34(1), 30-34. http://dx.doi.org/10.1016/ j.gerinurse.2012.07.001.

Thomas, C., \& Mackey, E. (2012). Influence of a clinical simulation elective on baccalaureate nursing student clinical confidence. J. Nurs. Educ., 51(4), 236-239. http://dx.doi.org/ 10.3928/01484834-20120224-03

Tosterud, R., Hedelin, B., \& Hall-Lord, M. L. (2013). Nursing students' perceptions of high- and low-fidelity simulation used as learning methods. Nurse Educ. Pract., 13(4), 262-270. http://dx.doi.org/10.1016/j.nepr.2013.02.002

Van Rensburg, G. H., \& Botma, Y. (2015). Bridging the gap between self-directed learning of nurse educators and effective student support. Curationis, 38(2), 1-7.

Weiner, G. M., Menghini, K., Zaichkin, J., Caid, A. E., Jacoby, C. J., \& Simon, W. M. (2011). Selfdirected versus traditional classroom training for neonatal resuscitation. Pediatrics, 127(4), 713-719. http://dx.doi.org/10.1542/pedS. 2010-2829 
White, A., Brannan, J., Long, J., \& Kruszka, K., (2013). Comparison of instructional methods: cognitive skills and confidence levels. Clin. Simul. Nurs., 9(10), e417-e423. http:// dx.doi.org/10.1016/j.ecns.2012.12.002

Williams, B., Boyle, M., Winship, C., Brightwell, R., Devenish, S., \& Munro, G. (2013). Examination of self-directed learning readiness of paramedic undergraduates: A multi-institutional study. Journal of Nursing Education and Practice, 3(2). http://dx.doi.org/10.5430/jnep.v3n2p102

Yang, G. F., \& Jiang, X. Y. (2014). Self-directed learning readiness and nursing competency among undergraduate nursing students in Fujian province of China. International Journal of Nursing Sciences, 1(3), 255-259.

Yuan, H. B., Williams, B. A., Fang, J. B., \& Pang, D. (2012). Chinese baccalaureate nursing students' readiness for self-directed learning. Nurse Education Today, 32(4), 427-431. http://dx.doi.org/10.1016/j.nedt.2012.01.002

\section{Copyrights}

Copyright for this article is retained by the author(s), with first publication rights granted to the journal.

This is an open-access article distributed under the terms and conditions of the Creative Commons Attribution license (http://creativecommons.org/licenses/by/4.0/). 